\title{
11. \\ Note sur la composition du nombre 47 par rapport aux vingt-troisièmes racines de l'unité. \\ (Par M. Cayley.)
}

II. Kummer a trouvé (Journal de M. Liouville t. XII, p. 208) que le nombre 47 peut être décomposé en onze facteurs qui se déduisent du suivant $\alpha^{11}+\alpha^{13}+\alpha^{8}+\alpha^{15}+\alpha^{7}+\alpha^{16}$, $\alpha$ désignant une racine $23^{\text {eme }}$ de l'unité, et on sait par la théorie générale qu'il doit $y$ avoir une puissance $47^{3 f}$ qui se décompose en vingt-deux facteurs. Le nombre $47^{3}$ peut se décomposer en deux facteurs formés avec les demi-périodes des racines; il était donc naturel d'essayer si le facteur $\left(\alpha^{11}+\alpha^{13}+\alpha^{8}+\alpha^{15}+\alpha^{7}+\alpha^{16}\right)^{3}$ pourrait se décomposer de même en deux facteurs, ce qui donnerait la décomposition de $47^{3}$ en vingt-deux facteurs. Mais on démontre très-facilement que cette décomposition n'est pas possible. En effet en posant $\alpha^{\lambda}=1$ ( $\lambda$ étant un nombre premier) et en faisant

$$
\boldsymbol{A}+\boldsymbol{B} \alpha+\cdots \boldsymbol{K} \alpha^{\lambda-1}=\left(\boldsymbol{a}+\boldsymbol{b} \alpha+\cdots \boldsymbol{k} \alpha^{\lambda-1}\right)\left(\boldsymbol{a}+b \alpha^{\lambda-1}+\cdots \boldsymbol{k} \alpha\right),
$$

on aura $\boldsymbol{A}=a^{2}+b^{2}+\cdots k^{2}$. Le nombre qui forme le premier membre peut se réduire au moyen de l'équation $1+\alpha+\cdots \alpha^{\lambda-1}=0$ à la forme $\boldsymbol{B}^{\prime} \alpha+\boldsymbol{C}^{\prime} \alpha^{2} \cdots+\boldsymbol{K}^{\prime} \alpha^{\lambda-1}$ et l'on aura

$$
\begin{gathered}
\boldsymbol{B}^{\prime} \alpha+\boldsymbol{C}^{\prime} \alpha^{2} \cdots+\boldsymbol{K}^{\prime} \alpha^{\lambda-1} \\
=\left(\boldsymbol{a}+\boldsymbol{b} \alpha+\cdots \boldsymbol{k} \alpha^{\lambda-1}\right)\left(\boldsymbol{a}+\boldsymbol{b} \alpha^{\lambda-1}+\cdots k \alpha\right)-\left(\boldsymbol{a}^{2}+\boldsymbol{b}^{2}+\cdots k^{2}\right)\left(1+\alpha+\cdots \alpha^{\lambda-1}\right),
\end{gathered}
$$

équation qui subsiste lorsqu'on $\mathrm{y}$ fait $\alpha=1$, ce qui donne

$$
\boldsymbol{B}^{\prime}+\boldsymbol{C}^{\prime}+\cdots \boldsymbol{K}^{\prime}=(a+b+\cdots k)^{2}-\lambda\left(a^{2}+b^{2}+\cdots k^{2}\right) ;
$$

or, la fonction qui forme le second membre, prise avec le signe négatif peut se mettre sous la forme $(a-b)^{2}+(a-c)^{2}+\left(b-c^{2}\right)+$ etc. donc la décomposition n'existe pas à moins que $\boldsymbol{B}^{\prime}+\boldsymbol{C}^{\prime}+\ldots \boldsymbol{K}^{\prime}$ ne soit négatif. Mais, en réduisant seulement au moyen de l'équation $\alpha^{23}-1=0$, on trouve la suivante

$$
\left(\alpha^{10}+\alpha^{13}+\alpha^{8}+\alpha^{15}+\alpha^{7}+\alpha^{16}\right)^{3}=
$$

$6+7 \alpha+7 \alpha^{2}+3 \alpha^{3}+6 \alpha^{4}+9 \alpha^{5}+6 \alpha^{6}+16 \alpha^{7}+15 \alpha^{8}+9 \alpha^{9}+18 \alpha^{10}+9 \alpha^{11}$ $+7 \alpha^{22}+7 \alpha^{21}+3 \alpha^{21}+6 \alpha^{19}+9 \alpha^{18}+6 \alpha^{17}+16 \alpha^{16}+15 \alpha^{15}+9 \alpha^{14}+18 \alpha^{13}+9 \alpha^{12}$ laquelle, en vertu de $1+\alpha+\cdots \alpha^{22}=0$, se réduit à

$$
\left.\begin{array}{r}
\alpha+\alpha^{2}-3 \alpha^{3}+3 \alpha^{5}+10 \alpha^{7}+9 \alpha^{8}+3 \alpha^{9}+12 \alpha^{10}+3 \alpha^{11} \\
+\alpha^{22}+\alpha^{21}-3 \alpha^{20}+3 \alpha^{18}+10 \alpha^{16}+9 \alpha^{15}+3 \alpha^{14}+12 \alpha^{13}+3 \alpha^{12}
\end{array}\right\}
$$

où la somme des coefficients est positive; donc la décomposition ne peut pas s'effectuer. On pourrait sans beaucoup de peine essayer de la même manière les nombres $f=2$ ou $f=3$, mais je ne sais pas si l'on a une idée quelconque de la grandeur du nombre $f$.

Londres, le 10 Mai 1857. 